ANNALES

POLONICI MATHEMATICI

$82.2(2003)$

\title{
Hamilton-Jacobi functional differential equations with unbounded delay
}

\author{
by AdAm NADOLski (Gdańsk)
}

\begin{abstract}
The Cauchy problem for nonlinear functional differential equations on the Haar pyramid is considered. The phase space for generalized solutions is constructed. An existence theorem is proved by using the method of successive approximations. The theory of characteristics and integral inequalities are used. Examples of phase spaces are given.
\end{abstract}

1. Introduction. For any metric spaces $U$ and $W$ we denote by $C(U, W)$ the class of all continuous functions defined on $U$ and taking values in $W$. Let $L\left([0, a], \mathbb{R}_{+}\right)$be the set of summable functions $\alpha:[0, a] \rightarrow \mathbb{R}_{+}=[0, \infty)$. Vectorial inequalities will be understood to hold componentwise.

Let $H$ denote the Haar pyramid

$$
H=\left\{(t, x)=\left(t, x_{1}, \ldots, x_{n}\right) \in \mathbb{R}^{1+n}: t \in[0, a],-b+h(t) \leq x \leq b-h(t)\right\}
$$

and

$$
E=(-\infty, 0] \times[-b, b] \subset \mathbb{R}^{1+n},
$$

where $b=\left(b_{1}, \ldots, b_{n}\right) \in \mathbb{R}_{+}^{n}$, and $h=\left(h_{1}, \ldots, h_{n}\right) \in C\left([0, a], \mathbb{R}_{+}^{n}\right), a>0$. We assume that $h$ is nondecreasing, $h(0)=0$ and $b>h(a)$.

Let $Y$ be a space of initial functions $w: E \rightarrow \mathbb{R}$. We assume that $Y$ is a linear space with a norm $\|\cdot\|_{Y}$ and that $\left(Y,\|\cdot\|_{Y}\right)$ is a Banach space. For $0<t \leq a$ we put $H_{t}=H \cap\left([0, t] \times \mathbb{R}^{n}\right)$. Let $\|\cdot\|_{t}$ be the supremum norm in $C\left(H_{t}, \mathbb{R}\right)$ or $C\left(H_{t}, \mathbb{R}^{n}\right)$. For each $t, 0<t \leq a$, we consider a space $X_{t}$ of functions $z: E \cup H_{t} \rightarrow \mathbb{R}$. We assume that $X_{t}$ is a linear space with a norm $\|\cdot\|_{X_{t}}$.

Write $X=X_{a}$ and $\|\cdot\|_{X}=\|\cdot\|_{X_{a}}$ and assume that $V: X \rightarrow C(H, \mathbb{R})$ is a given operator. Let $\Omega=H \times \mathbb{R} \times \mathbb{R}^{n}$ and assume that $f: \Omega \rightarrow \mathbb{R}$ and $\varphi: E \rightarrow \mathbb{R}$ are given functions. We consider the functional differential equation

$$
\partial_{t} z(t, x)=f\left(t, x,(V z)(t, x), \partial_{x} z(t, x)\right)
$$

2000 Mathematics Subject Classification: 35R10, 35D05.

Key words and phrases: unbounded delay, initial problems, Haar pyramid, generalized solutions. 
with the initial condition

$$
z(t, x)=\varphi(t, x) \quad \text { on } E
$$

where $\partial_{x} z=\left(\partial_{x_{1}} z, \ldots, \partial_{x_{n}} z\right)$.

Write $I[x]=\{t \in[0, a]:(t, x) \in H\}$ for $x \in[-b, b]$. We will consider generalized solutions of problem (1), (2). A function $v: E \cup H_{c} \rightarrow \mathbb{R}$, $0<c \leq a$, is a solution of (1), (2) provided

(i) $\left.v\right|_{H_{c}}$ is continuous and $\partial_{x} v(t, x)$ exists for $(t, x) \in H_{c}$,

(ii) $v(\cdot, x): I[x] \rightarrow \mathbb{R}$ is absolutely continuous for every $x \in[-b, b]$,

(iii) for every $x \in[-b, b]$ the function $v$ satisfies equation (1) for almost all $t \in I[x]$ and condition (2) holds.

Two different types of results on nonlinear first order partial differential or functional differential equations appear in the literature. The first type theorems deal with initial or initial boundary problems which are local with respect to $x$, while those of the second type are global with respect to the spatial variable. We are interested in results of the first type.

Numerous papers have been published concerning nonlinear first order partial differential or functional differential equations on the Haar pyramid. The following questions were considered: differential inequalities, uniqueness and continuous dependence for initial value problems $([2,6,11,16])$, difference inequalities and approximate solutions $([10,14])$, existence of classical or generalized solutions $([5,6,15,17])$. The papers $[1,3,4,18]$ initiated the investigations of functional differential inequalities generated by initial value problems on the Haar pyramid. Existence results for functional differential equations of the Volterra type with initial conditions can be found in [9]. The monograph [8] contains an exposition of recent developments in hyperbolic functional differential equations. All these problems have the following property: the initial set in the Cauchy problem is bounded.

It is the purpose of this paper to examine Hamilton-Jacobi functional differential equations with unbounded delay. We give sufficient conditions for the existence of generalized solutions of initial value problems. The set of axioms for phase spaces given here seems to be in final form for generalized or classical solutions.

Note that the theory of ordinary functional differential equations with unbounded delay has been described extensively in the monographs [7, 12]. Functional differential problems for quasi-linear hyperbolic systems with unbounded delay were considered in [13].

The paper is organized as follows. The set of axioms for phase spaces is formulated in Section 2. The notion of characteristics for problem (1), (2) and their properties are given in the next section. Then the Cauchy problem is transformed into a system of integral functional equations. This system is 
solved in Sections 5 and 6 by the method of successive approximations. The main existence result and a theorem on continuous dependence of solutions on initial functions is presented in the last section of the paper.

2. Function spaces. Let $C^{I}\left(H_{t}, \mathbb{R}\right), 0<t \leq a$, be the set of all continuous functions $w: H_{t} \rightarrow \mathbb{R}$ such that the derivatives $\left(\partial_{x_{1}} w, \ldots, \partial_{x_{n}} w\right)=\partial_{x} w$ exist and $\partial_{x} w \in C\left(H_{t}, \mathbb{R}^{n}\right)$. For $w \in C^{I}\left(H_{t}, \mathbb{R}\right)$ we put

$$
\|w\|_{t}^{I}=\|w\|_{t}+\max \left\{\left\|\partial_{x} w(\tau, y)\right\|:(\tau, y) \in H_{t}\right\} .
$$

We denote by $C^{I+L}\left(H_{t}, R\right), 0<t \leq a$, the set of all $w \in C^{I}\left(H_{t}, \mathbb{R}\right)$ such that

$\left.\operatorname{Lip} \partial_{x} w\right|_{H_{t}}$

$$
=\sup \left\{\frac{\left\|\partial_{x} w(\tau, y)-\partial_{x} w(\tau, \bar{y})\right\|}{\|y-\bar{y}\|}:(\tau, y),(\tau, \bar{y}) \in H_{t}, y \neq \bar{y}\right\}<\infty .
$$

For $w \in C^{I+L}\left(H_{t}, \mathbb{R}\right)$ we put

$$
\|w\|_{t}^{I+L}=\|w\|_{t}^{I}+\left.\operatorname{Lip} \partial_{x} w\right|_{H_{t}} .
$$

Assumption $\mathrm{H}[X]$. Suppose that $\left(Y,\|\cdot\|_{Y}\right)$ and $\left(X_{t},\|\cdot\|_{X_{t}}\right), 0<t \leq a$, are Banach spaces and

1) if $z: E \cup H_{t} \rightarrow \mathbb{R}$ and $\left.z\right|_{E} \in Y,\left.z\right|_{H_{t}} \in C\left(H_{t}, \mathbb{R}\right)$ then $z \in X_{t}$ and

$$
\|z\|_{X_{t}} \leq\left\|\left.z\right|_{E}\right\|_{Y}+\left\|\left.z\right|_{H_{t}}\right\|_{t},
$$

2) if $w \in Y$ then $|w(0, x)| \leq\|w\|_{Y}$ for $x \in[-b, b]$,

3) the linear subspaces $X_{t}^{I} \subset X_{t}, 0<t \leq a$, and $Y^{I} \subset Y$ are such that

(i) $X_{t}^{I}$ endowed with the norm $\|\cdot\|_{X_{t}^{I}}$ and $Y^{I}$ endowed with the norm $\|\cdot\|_{Y^{I}}$ are Banach spaces,

(ii) if $z: E \cup H_{t} \rightarrow \mathbb{R}$ and $\left.z\right|_{E} \in Y^{I},\left.z\right|_{H_{t}} \in C^{I}\left(H_{t}, \mathbb{R}\right)$ then $z \in X_{t}^{I}$ and

$$
\|z\|_{X_{t}^{I}} \leq\left\|\left.z\right|_{E}\right\|_{Y^{I}}+\left\|\left.z\right|_{H_{t}}\right\|_{t}^{I},
$$

(iii) if $w \in Y^{I}$ then $\partial_{x} w(0, x)$ exists for $x \in[-b, b]$ and $|w(0, x)|+$ $\left\|\partial_{x} w(0, x)\right\| \leq\|w\|_{Y^{I}}$ for $x \in[-b, b]$,

4) the linear subspaces $X_{t}^{I+L} \subset X_{t}^{I}, 0<t \leq a$, and $Y^{I+L} \subset Y^{I}$ are such that

(i) $X_{t}^{I+L}$ endowed with the norm $\|\cdot\|_{X_{t}^{I+L}}$ and $Y^{I+L}$ endowed with the norm $\|\cdot\|_{Y^{I+L}}$ are Banach spaces,

(ii) if $z: E \cup H_{t} \rightarrow \mathbb{R}$ and $\left.z\right|_{E} \in Y^{I+L},\left.z\right|_{H_{t}} \in C^{I+L}\left(H_{t}, R\right)$ then $z \in X_{t}^{I+L}$ and

$$
\|z\|_{X_{t}^{I+L}} \leq\left\|\left.z\right|_{E}\right\|_{Y^{I+L}}+\left\|\left.z\right|_{H_{t}}\right\|_{t}^{I+L} .
$$


Suppose that $\varphi \in Y^{I+L}$ and $0<c \leq a, d=\left(d_{0}, d_{1}, d_{2}\right) \in \mathbb{R}_{+}^{3}, \lambda=$ $\left(\lambda_{0}, \lambda_{1}\right), \lambda_{0}, \lambda_{1} \in L\left([0, c], \mathbb{R}_{+}\right)$. We denote by $C_{\varphi . c}^{I+L}[d, \lambda]$ the class of all functions $z: E \cup H_{c} \rightarrow \mathbb{R}$ such that

(i) $z(t, x)=\varphi(t, x)$ on $E$,

(ii) $\left.z\right|_{H_{c}}$ is continuous and $|z(t, x)| \leq d_{0}$ on $H_{c}$,

(iii) $\partial_{x} z(t, x)$ exists for $(t, x) \in H_{c}$ and $\left\|\partial_{x} z(t, x)\right\| \leq d_{1}$ on $H_{c}$,

(iv) for $(t, x),(\bar{t}, \bar{x}),(\bar{t}, x) \in H_{c}$ we have

$$
\begin{aligned}
|z(t, x)-z(\bar{t}, x)| & \leq\left|\int_{t}^{\bar{t}} \lambda_{0}(\xi) d \xi\right|, \\
\left\|\partial_{x} z(t, x)-\partial_{x} z(\bar{t}, \bar{x})\right\| & \leq\left|\int_{t}^{\bar{t}} \lambda_{1}(\xi) d \xi\right|+d_{2}\|x-\bar{x}\| .
\end{aligned}
$$

Let $s=\left(s_{0}, s_{1}\right) \in \mathbb{R}_{+}^{2}$ and $\mu \in L\left([0, c], \mathbb{R}_{+}\right)$. We denote by $C_{c}^{L}[s, \mu]$ the class of all functions $v: H_{c} \rightarrow \mathbb{R}^{n}$ such that

(i) $v \in C\left(H_{c}, \mathbb{R}^{n}\right)$ and $\|v(t, x)\| \leq s_{0}$ on $H_{c}$,

(ii) for $(t, x),(\bar{t}, \bar{x}) \in H_{c}$ we have

$$
\|v(t, x)-v(\bar{t}, \bar{x})\| \leq\left|\int_{t}^{\bar{t}} \mu(\xi) d \xi\right|+s_{1}\|x-\bar{x}\| .
$$

Under suitable assumptions on the given functions we will prove that there exists a solution $v$ of problem (1), (2) such that $v \in C_{\varphi . c}^{I+L}[d, \lambda]$ and $u \in$ $C_{c}^{L}[s, \mu]$, where $u=\left.\partial_{x} v\right|_{H_{c}}$.

Now we formulate assumptions on the operator $V$.

Assumption $\mathrm{H}[V]$. Suppose that $V: X \rightarrow C(H, \mathbb{R})$ and

1) there is $L_{0} \in \mathbb{R}_{+}$such that for $z, \bar{z} \in X$ we have

$$
\|V z-V \bar{z}\|_{t} \leq L_{0}\|z-\bar{z}\|_{X_{t}}
$$

2) if $z \in X_{t}^{I}, 0<t \leq a$, then $V z \in C^{I}\left(H_{t}, \mathbb{R}\right)$ and there is $L \in \mathbb{R}_{+}$such that for $z, \bar{z} \in X_{t}^{I}$ we have

$$
\|V z-V \bar{z}\|_{t}^{I} \leq L\|z-\bar{z}\|_{X_{t}^{I}}
$$

3) if $z \in C^{I+L}\left(H_{t}, \mathbb{R}\right), 0<t \leq a$, then $V z \in C^{I+L}\left(H_{t}, \mathbb{R}\right)$ and there are $M, C \in \mathbb{R}_{+}$independent of $z$ such that

$$
\|V z\|_{t}^{I+L} \leq M\|z\|_{X_{t}^{I+L}}+C
$$

REMARK 2.1. If Assumption $\mathrm{H}[V]$ is satisfied then the operator $V$ satisfies the following Volterra condition: if $(t, x) \in H, z, \bar{z} \in X$ and $z(\tau, y)=$ $\bar{z}(\tau, y)$ for $(\tau, y) \in E \cup H_{t}$ then $(V z)(t, x)=(V \bar{z})(t, x)$. 
Lemma 2.2. Suppose that Assumption $\mathrm{H}[V]$ is satisfied and $\theta \in X$ is given by $\theta(\tau, y)=0$ for $(\tau, y) \in E \cup H$.

If $z \in X, 0<t \leq a$, then

$$
\|V z\|_{t} \leq L_{0}\|z\|_{X_{t}}+A, \text { where } A \geq\|V \theta\|_{t} .
$$

If $z \in X_{t}^{I}, 0<t \leq a$, then

$$
\|V z\|_{t}^{I} \leq L\|z\|_{X_{t}^{L}}+B, \quad \text { where } \quad B \geq\|V \theta\|_{t}^{I} .
$$

We omit the proof of the lemma.

Now we give examples of spaces of initial functions.

EXAMPLE 2.3. Let $\gamma:(-\infty, 0] \rightarrow(0, \infty)$ be a given function with $\gamma(0)=1$. Let $Y$ be the space of all continuous functions $w: E \rightarrow \mathbb{R}$ such that

$$
\|w\|_{Y}=\sup \left\{\frac{|w(t, x)|}{\gamma(t)}:(t, x) \in E\right\}<\infty
$$

Denote by $Y^{I}$ the class of all $w \in Y$ such that $\left(\partial_{x_{1}} w, \ldots, \partial_{x_{n}} w\right)=\partial_{x} w$ exists on $E, \partial_{x} w \in C\left(E, \mathbb{R}^{n}\right)$ and

$$
\left\|\partial_{x} w\right\|_{Y}=\sup \left\{\frac{\left\|\partial_{x} w(t, x)\right\|}{\gamma(t)}:(t, x) \in E\right\}<\infty .
$$

For $w \in Y^{I}$ we put

$$
\|w\|_{Y^{I}}=\|w\|_{Y}+\left\|\partial_{x} w\right\|_{Y}
$$

Let $Y^{I+L}$ be the space of all $w \in Y^{I}$ which satisfy $\operatorname{Lip}\left[\partial_{x} w\right]_{\gamma}<\infty$, where

$$
\operatorname{Lip}\left[\partial_{x} w\right]_{\gamma}=\sup \left\{\frac{\left\|\partial_{x} w(t, x)-\partial_{x} w(t, \bar{x})\right\|}{\gamma(t)\|x-\bar{x}\|}:(t, x),(t, \bar{x}) \in E, x \neq \bar{x}\right\} .
$$

We define the norm in $Y^{I+L}$ by

$$
\|w\|_{Y^{I+L}}=\|w\|_{Y^{L}}+\operatorname{Lip}\left[\partial_{x} w\right]_{\gamma} .
$$

Example 2.4. Fix $p \geq 1$. Denote by $\tilde{Y}$ the class of all functions $w \in$ $C(E, \mathbb{R})$ such that for $x \in[-b, b]$ we have

Write

$$
\int_{-\infty}^{0}|w(\tau, x)|^{p} d \tau<\infty .
$$

$$
\begin{aligned}
\|w\|_{\tilde{Y}}= & \max \{|w(0, x)|: x \in[-b, b]\} \\
& +\sup \left\{\left(\int_{-\infty}^{0}|w(\tau, x)|^{p} d \tau\right)^{1 / p}: x \in[-b, b]\right\} .
\end{aligned}
$$

Denote by $Y$ the closure of $\widetilde{Y}$ with the above norm.

Let $Y^{I} \subset Y$ be the set of all $w \in Y$ such that the derivative $\partial_{x} w(t, x)$ exists on $E$ and 
(i) $\partial_{x} w$ is continuous on $\{0\} \times[-b, b]$,

(ii) for $x \in[-b, b]$ we have

$$
\left\|\partial_{x} w\right\|_{p}=\sup \left\{\left(\int_{-\infty}^{0}\left\|\partial_{x} w(\tau, x)\right\|^{p} d \tau\right)^{1 / p}: x \in[-b, b]\right\}<\infty
$$

Write

$$
\|w\|_{Y^{I}}=\|w\|_{Y}+\left\|\partial_{x} w\right\|_{p}+\max \left\{\left\|\partial_{x} w(0, x)\right\|: x \in[-b, b]\right\} .
$$

Let $Y^{I+L}$ be the set of all $w \in Y^{I}$ such that Lip $\left[\partial_{x} w\right]_{p}<\infty$, where $\operatorname{Lip}\left[\partial_{x} w\right]_{p}$

$$
=\sup \left\{\frac{\left(\int_{-\infty}^{0} \| \partial_{x} w(\tau, x)-\partial_{x} w\left(\tau, \bar{x} \|^{p} d \tau\right)^{1 / p}\right.}{\|x-\bar{x}\|^{-1}}:(t, x),(t, \bar{x}) \in E, x \neq \bar{x}\right\} .
$$

We define the norm in $Y^{I+L}$ by

$$
\|w\|_{Y^{I+L}}=\|w\|_{Y^{I}}+\operatorname{Lip}\left[\partial_{x} w\right]_{p} .
$$

EXAMPLE 2.5. Let $\widetilde{Y}$ be the space of all functions $w \in C(E, \mathbb{R})$ which are bounded and

$$
\|w\|_{*}=\sup \left\{\int_{-(n+1)}^{-n}|w(\tau, x)| d \tau: x \in[-b, b], n \in \mathbb{N}\right\}<\infty,
$$

where $\mathbb{N}$ is the set of natural numbers. For $w \in \tilde{Y}$ we put

$$
\|w\|_{\widetilde{Y}}=\|w\|_{*}+\max \{|w(0, x)|: x \in[-b, b]\} .
$$

Let $Y$ be the closure of $\tilde{Y}$ with the above norm.

Denote by $Y^{I}$ the class of all $w \in Y$ such that the derivative $\partial_{x} w$ exists on $E, \partial_{x} w$ is continuous for almost all $t \in(-\infty, 0)$ and

$$
\left\|\partial_{x} w\right\|_{*}=\sup \left\{\int_{-(n+1)}^{-n}\left\|\partial_{x} w(\tau, x)\right\| d \tau: x \in[-b, b], n \in \mathbb{N}\right\}<\infty .
$$

For $w \in \tilde{Y}^{I}$ we put

$$
\|w\|_{Y^{I}}=\|w\|_{Y}+\left\|\partial_{x} w\right\|_{*}+\max \left\{\left\|\partial_{x} w(0, x)\right\|: x \in[-b, b]\right\} .
$$

Let $Y^{I+L}$ be the space of all $w \in Y^{I}$ such that $\operatorname{Lip}\left[\partial_{x} w\right]_{*}<\infty$, where $\operatorname{Lip}\left[\partial_{x} w\right]_{*}=\sup \left\{\frac{\int_{-(n+1)}^{-n}\left\|\partial_{x} w(\tau, x)-\partial_{x} w(\tau, \bar{x})\right\| d \tau}{\|x-\bar{x}\|}: x, \bar{x} \in[-b, b], n \in \mathbb{N}\right\}$.

We define the norm in $Y^{I+L}$ by

$$
\|w\|_{Y^{I+L}}=\|w\|_{Y^{I}}+\operatorname{Lip}\left[\partial_{x} w\right]_{*} .
$$

It is easy to find suitable spaces $X_{t}, 0<t \leq a$, corresponding to the above sets of initial functions. 
3. Characteristics of nonlinear equations. We start with the assumptions on $f$.

Assumption $\mathrm{H}\left[\partial_{q} f\right]$. Suppose that the function $f: \Omega \rightarrow \mathbb{R}$ of the variables $(t, x, p, q), q=\left(q_{1}, \ldots, q_{n}\right)$, is such that

$1)$ the derivative $\left(\partial_{q_{1}} f, \ldots, \partial_{q_{n}} f\right)=\partial_{q} f$ exists on $\Omega$ and $\partial_{q} f(\cdot, x, p, q)$ : $I[x] \rightarrow \mathbb{R}^{n}$ is measurable for $(x, p, q) \in[-b, b] \times \mathbb{R} \times \mathbb{R}^{n}$,

2) there is $\gamma=\left(\gamma_{1}, \ldots, \gamma_{n}\right) \in L\left([0, a], \mathbb{R}_{+}^{n}\right)$ such that

$$
\left|\partial_{q_{i}} f(t, x, p, q)\right| \leq \gamma_{i}(t) \quad \text { on } \Omega \text { for } 1 \leq i \leq n,
$$

3) there is $\beta \in L\left([0, a], \mathbb{R}_{+}\right)$such that

$$
\left\|\partial_{q} f(t, x, p, q)-\partial_{q} f(t, \bar{x}, \bar{p}, \bar{q})\right\| \leq \beta(t)[\|x-\bar{x}\|+|p-\bar{p}|+\|q-\bar{q}\|]
$$

on $\Omega$,

4) for $t \in[0, a]$ we have

$$
h(t)=\int_{0}^{t} \gamma(\xi) d \xi
$$

Now we define the notion of characteristics for equation (1). Suppose that $\varphi \in Y^{I+L}$ and $z \in C_{\varphi \cdot c}^{I+L}[d, \lambda], u \in C_{c}^{L}[s, \mu]$. We consider the Cauchy problem

$$
\eta^{\prime}(\tau)=-\partial_{q} f(\tau, \eta(\tau),(V z)(\tau, \eta(\tau)), u(\tau, \eta(\tau))), \quad \eta(t)=x,
$$

where $(t, x) \in H_{c}$. Let

$$
g[z, u](\cdot, t, x)=\left(g_{1}[z, u](\cdot, t, x), \ldots, g_{n}[z, u](\cdot, t, x)\right)
$$

be a Carathéodory solution of problem (3). The function $g[z, u]$ is the characteristic of equation (1) corresponding to $[z, u]$.

Lemma 3.1. Suppose that Assumptions $\mathrm{H}[X], \mathrm{H}[V], \mathrm{H}\left[\partial_{q} f\right]$ are satisfied and

$$
\varphi, \bar{\varphi} \in Y^{I+L}, \quad z \in C_{\varphi . c}^{I+L}[d, \lambda], \quad \bar{z} \in C_{\bar{\varphi} . c}^{I+L}[d, \lambda], \quad u, \bar{u} \in C_{c}^{L}[s, \mu],
$$

where $0<c \leq a$. Then the characteristics $g[z, u](\cdot, t, x)$ and $g[\bar{z}, \bar{u}](\cdot, t, x)$ are defined on intervals $[0, \kappa(t, x)]$ and $[0, \bar{\kappa}(t, x)]$ such that for $\xi=\kappa(t, x)$, $\bar{\xi}=\bar{\kappa}(t, x)$ we have

$$
(\xi, g[z, u](\xi, t, x)) \in \partial H_{c}, \quad(\bar{\xi}, g[\bar{z}, \bar{u}](\bar{\xi}, t, x)) \in \partial H_{c}
$$

where $\partial H_{c}$ is the boundary of $H_{c}$. Moreover the solutions are unique and we have the estimates

(4) $\|g[z, u](\tau, t, x)-g[z, u](\tau, \bar{t}, \bar{x})\| \leq \beta^{\star}(\tau, t)\left[\|x-\bar{x}\|+\left|\int_{t}^{\bar{t}}\|\gamma(\xi)\| d \xi\right|\right]$ 
for $\tau \in[0, \min \{\kappa(t, x), \kappa(\bar{t}, \bar{x})\}]$ and

$$
\begin{aligned}
& \|g[z, u](\tau, t, x)-g[\bar{z}, \bar{u}](\tau, t, x)\| \\
& \quad \leq \beta^{\star}(\tau, t)\left|\int_{t}^{\tau} \beta(\xi)\left[L_{0}\|z-\bar{z}\|_{X_{\xi}}+\|u-\bar{u}\|_{\xi}\right] d \xi\right|
\end{aligned}
$$

for $\tau \in[0, \min \{\kappa(t, x), \bar{\kappa}(t, x)\}]$, where

$$
\beta^{\star}(\tau, t)=\exp \left[\bar{d}\left|\int_{\tau}^{t} \beta(\xi) d \xi\right|\right], \quad \bar{d}=1+B+s_{1}+L\left(\|\varphi\|_{Y^{I}}+d_{0}+d_{1}\right) .
$$

Proof. The existence and uniqueness of the solution of (3) follows from classical theorems. Note that the right-hand side of the differential system satisfies the Carathéodory assumptions, and the Lipschitz condition $\left\|\partial_{q} f(\tau, y,(V z)(\tau, y), u(\tau, y))-\partial_{q} f(\tau, \bar{y},(V z)(\tau, \bar{y}), u(\tau, \bar{y}))\right\| \leq \widetilde{\beta}(\tau)\|y-\bar{y}\|$ holds on $H_{c}$, where

$$
\widetilde{\beta}(\tau)=\beta(\tau)\left[1+B+L\|z\|_{X_{t}^{I}}+s_{1}\right] .
$$

If we transform problem (3) into an integral equation, then by Assumptions $\mathrm{H}[V]$ and $\mathrm{H}\left[\partial_{q} f\right]$ we have the integral inequality

$$
\begin{aligned}
\|g[z, u](\tau, t, x)-g[z, u](\tau, \bar{t}, \bar{x})\| & \leq\|x-\bar{x}\|+\left|\int_{t}^{\bar{t}}\|\gamma(\xi)\| d \xi\right| \\
& +\bar{d}\left|\int_{\tau}^{t} \beta(\xi)\|g[z, u](\xi, t, x)-g[z, u](x, \bar{t}, \bar{x})\| d \xi\right|
\end{aligned}
$$

for $\tau \in[0, \min \{\kappa(t, x), \kappa(\bar{t}, \bar{x})]$. Now we obtain (4) by the Gronwall inequality.

Analogously by Assumptions $\mathrm{H}[V]$ and $\mathrm{H}\left[\partial_{q} f\right]$ we get

$$
\begin{aligned}
\|g[z, u](\tau, t, x)-g[\bar{z}, \bar{u}](\tau, t, x)\| & \leq\left|\int_{t}^{\tau} \beta(\xi)\left[L_{0}\|z-\bar{z}\|_{X_{\xi}}+\|u-\bar{u}\|_{\xi}\right] d \xi\right| \\
& +\bar{d}\left|\int_{t}^{\tau} \beta(\xi)\|g[z, u](\xi, t, x)-g[\bar{z}, \bar{u}](\xi, t, x)\| d \xi\right|
\end{aligned}
$$

for $\tau \in[0, \min \{\kappa(t, x), \bar{\kappa}(t, x)]$. Thus (5) follows from the Gronwall inequality. This proves Lemma 3.1.

4. Functional integral operators. Now we formulate further assumptions on $f$ and $\varphi$.

Assumption $\mathrm{H}[f, \varphi]$. Suppose that Assumption $\mathrm{H}\left[\partial_{q} f\right]$ is satisfied and

$1)$ there is $\alpha \in L\left([0, a], \mathbb{R}_{+}\right)$such that $|f(t, x, p, q)| \leq \alpha(t)$ on $\Omega$, 
2) the derivatives $\left(\partial_{x_{1}} f, \ldots, \partial_{x_{n}} f\right)=\partial_{x} f$ and $\partial_{p} f$ exist on $\Omega$ and for every $(x, p, q) \in[-b, b] \times \mathbb{R} \times \mathbb{R}^{n}$ the functions

$$
\partial_{x} f(\cdot, x, p, q): I[x] \rightarrow \mathbb{R}^{n}, \quad \partial_{p} f(\cdot, x, p, q): I[x] \rightarrow \mathbb{R}
$$

are measurable,

$3)$ there is $\widetilde{\gamma} \in L\left([0, a], \mathbb{R}_{+}\right)$such that

$$
\left\|\partial_{x} f(t, x, p, q)\right\| \leq \widetilde{\gamma}(t), \quad\left|\partial_{p} f(t, x, p, q)\right| \leq \widetilde{\gamma}(t)
$$

on $\Omega$ and the expressions

$$
\left\|\partial_{x} f(t, x, p, q)-\partial_{x} f(t, \bar{x}, \bar{p}, \bar{q})\right\|, \quad\left|\partial_{p} f(t, x, p, q)-\partial_{p} f(t, \bar{x}, \bar{p}, \bar{q})\right|
$$

are bounded from above by

$$
\beta(t)[\|x-\bar{x}\|+|p-\bar{p}|+\|q-\bar{q}\|],
$$

4) the function $\varphi(0, \cdot):[-b, b] \rightarrow \mathbb{R}$ is continuous, the derivative $\partial_{x} \varphi(0, \cdot)$ $=\left(\partial_{x_{1}} \varphi(0, \cdot), \ldots \partial_{x_{n}} \varphi(0, \cdot)\right)$ exists on $[-b, b]$ and

$$
\begin{gathered}
|\varphi(0, x)| \leq r_{0}, \quad\left\|\partial_{x} \varphi(0, x)\right\| \leq r_{1}, \\
\left\|\partial_{x} \varphi(0, x)-\partial_{x} \varphi(0, \bar{x})\right\| \leq r_{2}\|x-\bar{x}\|,
\end{gathered}
$$

for $x, \bar{x} \in[-b, b]$.

REMARK 4.1. For simplicity of notations, we have assumed the same estimate for $\partial_{x} f$ and $\partial_{p} f$. We have also assumed the Lipschitz condition for $\partial_{q} f, \partial_{x} f, \partial_{p} f$ with the same constant.

Now we find a system of integral functional equations which are generated by (1), (2). Write

$$
\begin{aligned}
P[z, u] & (\tau, t, x) \\
& =(\tau, g[z, u](\tau, t, x),(V z)(\tau, g[z, u](\tau, t, x)), u(\tau, g[z, u](\tau, t, x))) .
\end{aligned}
$$

Given $\varphi \in Y^{I+L}, z \in C_{\varphi . c}^{I+L}[d, \lambda], u \in C_{c}^{L}[s, \mu]$, we define

$$
\begin{aligned}
F[z, u](t, x)= & \varphi(0, g[z, u](0, t, x))+\int_{0}^{t} f(P[z, u](\tau, t, x)) d \tau \\
& -\sum_{k=1}^{n} \int_{0}^{t} \partial_{q_{k}} f(P[z, u](\tau, t, x)) u_{k}(\tau, g[z, u](\tau, t, x)) d \tau
\end{aligned}
$$

and

$$
\begin{aligned}
& G_{i}[z, u](t, x)=\partial_{x_{i}} \varphi(0, g[z, u](0, t, x))+\int_{0}^{t} \partial_{x_{i}} f(P[z, u](\tau, t, x)) d \tau \\
& \quad+\int_{0}^{t} \partial_{p} f(P[z, u](\tau, t, x)) \partial_{x_{i}}(V z)(\tau, g[z, u](\tau, t, x)) d \tau, \quad i=1, \ldots, n .
\end{aligned}
$$


Moreover we put

$$
G[z, u]=\left(G_{1}[z, u], \ldots, G_{n}[z, u]\right) .
$$

We will consider the following system of functional integral equations:

$$
z=F[z, u], \quad u=G[z, u], \quad z=\varphi \quad \text { on } E
$$

and

$$
g[z, u](\tau, t, x)=x+\int_{\tau}^{t} \partial_{q} f(P[z, u](\xi, t, x)) d \xi .
$$

The above system is obtained in the following way. We introduce first an additional unknown function $u=\left(u_{1}, \ldots, u_{n}\right)=\partial_{x} z$ in (1). Then we consider the following linearization of (1) with respect to $u$ :

$$
\partial_{t} z(t, x)=f(U)+\sum_{j=1}^{n} \partial_{q_{j}} f(U)\left(\partial_{x_{j}} z(t, x)-u_{j}(t, x)\right),
$$

where $U=(t, x,(V z)(t, x), u(t, x))$. By (1) we get the differential equations for the unknown function $u$ :

$$
\begin{aligned}
\partial_{t} u_{i}(t, x)= & \partial_{x_{i}} f(U)+\partial_{p} f(U) \partial_{x_{i}}(V z)(t, x) \\
& +\sum_{j=1}^{n} \partial_{q_{j}} f(U) \partial_{x_{j}} u_{i}(t, x), \quad 1 \leq i \leq n .
\end{aligned}
$$

We assume the following initial condition for $u$ :

$$
u(0, x)=\partial_{x} \varphi(0, x) \quad \text { for } x \in[-b, b] .
$$

Note that the quasi-linear system (11), (12) has the following property: the differential equations of the characteristics for (11) and (12) are the same and they have the form (3). Considering (11) and (12) along the characteristic $g[z, u](\cdot, t, x)$, we obtain

$$
\begin{aligned}
\frac{d}{d \tau} z(\tau, g[z, u](\tau, t, x))= & f(P[z, u](\tau, t, x)) \\
& -\sum_{j=1}^{n} \partial_{q_{j}} f(P[z, u](\tau, t, x)) u_{j}(\tau, g[z, u](\tau, t, x)),
\end{aligned}
$$

and

$$
\begin{aligned}
& \frac{d}{d \tau} u_{i}(\tau, g[z, u](\tau, t, x))=\partial_{x_{i}} f(P[z, u](\tau, t, x)) \\
& \quad+\partial_{p} f(P[z, u](\tau, t, x)) \partial_{x_{i}}(V z)(\tau, g[z, u](\tau, t, x)), \quad 1 \leq i \leq n .
\end{aligned}
$$

By integrating (14), (15) on $[0, t]$ with respect to $\tau$ and using the initial conditions (2), (13) we get (9), (10).

The proof of the existence of a solution of (9), (10) will be based on the following method of successive approximations. We define $z^{(m)}: E \cup H_{c} \rightarrow \mathbb{R}$ 
and $u^{(m)}: H_{c} \rightarrow \mathbb{R}^{n}$ in the following way. We put first

$$
\begin{aligned}
& z^{(0)}(t, x)= \begin{cases}\varphi(t, x) & \text { on } E, \\
\varphi(0, x) & \text { on } H_{c},\end{cases} \\
& u^{(0)}(t, x)=\partial_{x} \varphi(0, x) \text { on } H_{c} .
\end{aligned}
$$

If $z^{(m)}: E \cup H_{c} \rightarrow \mathbb{R}$ and $u^{(m)}: H_{c} \rightarrow \mathbb{R}^{n}$ are known, then

(i) $u^{(m+1)}$ is a solution of the equation

$$
u=G^{(m)}[u]
$$

where $G^{(m)}=\left(G_{1}^{(m)}, \ldots, G_{n}^{(m)}\right)=G\left[z^{(m)}, u\right]$,

(ii) $z^{(m+1)}$ is given by

$$
z^{(m+1)}(t, x)= \begin{cases}\varphi(t, x) & \text { on } E, \\ F\left[z^{(m)}, u^{(m+1)}\right](t, x) & \text { on } H_{c} .\end{cases}
$$

The problem of the existence of the sequence $\left\{z^{(m)}\right\},\left\{u^{(m)}\right\}$ is the main difficulty in our method.

5. Existence of the sequence of successive approximations. In this section we prove that the sequences $\left\{z^{(m)}\right\}$ and $\left\{u^{(m)}\right\}$ are defined on $E \cup H_{c}$ and $H_{c}$ respectively provided $c \in(0, a]$ is sufficiently small.

TheOREM 5.1. If Assumptions $\mathrm{H}[X], \mathrm{H}[V], \mathrm{H}[f, \varphi]$ are satisfied and $\varphi \in$ $Y^{I+L}$ then there are constants $\left(d_{0}, d_{1}, d_{2}\right)=d \in \mathbb{R}_{+}^{3},\left(s_{0}, s_{1}\right)=s \in \mathbb{R}_{+}^{2}$, $c \in(0, a]$ and functions $\mu, \lambda=\left(\lambda_{0}, \lambda_{1}\right), \mu, \lambda_{0}, \lambda_{1} \in L\left([0, c], \mathbb{R}_{+}\right)$such that for any $m \geq 0$ we have

$\left(\mathrm{I}_{m}\right)$ there exist $z^{(m)}: E \cup H_{c} \rightarrow \mathbb{R}$ and $u^{(m)}: H_{c} \rightarrow \mathbb{R}^{n}$ and

$$
z^{(m)} \in C_{\varphi . c}^{I+L}[d, \lambda], \quad u^{(m)} \in C_{c}^{L}[s, \mu],
$$

$\left(\mathrm{II}_{m}\right) \partial_{x} z^{(m)}(t, x)=u^{(m)}(t, x)$ on $H_{c}$.

Proof. The proof is divided into a sequence of steps.

I. We begin by defining some functions and constants. Write

$$
\begin{aligned}
P & =1+B+L\left(\|\varphi\|_{Y^{I}}+d_{0}+d_{1}\right), \\
\widetilde{P} & =C+M\left(\|\varphi\|_{Y^{I+L}}+d_{0}+d_{1}+d_{2}\right), \\
\Gamma(t) & =\beta^{\star}(0, t)\left\{r_{2}+P \bar{d} \int_{0}^{t} \beta(\xi) d \xi+\widetilde{P} \int_{0}^{t} \widetilde{\gamma}(\xi) d \xi\right\}, \\
\widetilde{\Gamma}(t) & =\beta^{\star}(0, t)\left\{r_{1}+\int_{0}^{t}\left[P \widetilde{\gamma}(\xi)+2 s_{1}\|\gamma(\xi)\|+s_{0} \bar{d} \beta(\xi)\right] d \xi\right\},
\end{aligned}
$$


where $\beta^{\star}$ is given by (6). Suppose that the constants $d=\left(d_{0}, d_{1}, d_{2}\right), s=$ $\left(s_{0}, s_{1}\right), c \in(0, a]$ and the functions $\lambda=\left(\lambda_{0}, \lambda_{1}\right), \mu$ satisfy the conditions

$$
\begin{aligned}
& s_{0}=d_{1} \geq r_{1}+P \int_{0}^{c} \widetilde{\gamma}(\xi) d \xi, \quad s_{1}=d_{2} \geq \Gamma(c), \\
& \mu(t)=\lambda_{1}(t)=\Gamma(c)\|\gamma(t)\|+P \widetilde{\gamma}(t), \\
& d_{0} \geq r_{0}+\int_{0}^{c}\left[\alpha(\xi)+s_{0}\|\gamma(\xi)\|\right] d \xi, \\
& \lambda_{0}(t)=\widetilde{\Gamma}(c)\|\gamma(t)\|+\alpha(t) .
\end{aligned}
$$

Note that if we assume that $s_{0}=d_{1}>r_{1}, s_{1}=d_{2}>r_{2}$ and $d_{0}>r_{0}$ then there is $c \in(0, a]$ such that conditions (20), (22) are satisfied.

II. We prove $\left(\mathrm{I}_{m}\right)$ and $\left(\mathrm{II}_{m}\right)$ by induction. It follows from (16), (17) that $\left(\mathrm{I}_{0}\right)$ and $\left(\mathrm{II}_{0}\right)$ are satisfied. Supposing now that $\left(\mathrm{I}_{m}\right)$ and $\left(\mathrm{II}_{m}\right)$ hold for given $m \geq 0$, we will prove that there exists $u^{(m+1)}: H_{c} \rightarrow \mathbb{R}^{n}$ and $u^{(m+1)} \in C_{c}^{L}[s, \mu]$. We claim that

$$
G^{(m)}: C_{c}^{L}[s, \mu] \rightarrow C_{c}^{L}[s, \mu] .
$$

It follows from Assumptions $\mathrm{H}[V]$ and $\mathrm{H}[f, \varphi]$ that

$$
\left\|G^{(m)}[u](t, x)\right\| \leq r_{1}+P \int_{0}^{t} \widetilde{\gamma}(\xi) d \xi \leq s_{0} \quad \text { on } H_{c} .
$$

Assumptions $\mathrm{H}[V]$ and $\mathrm{H}[X]$ imply the estimates

$$
\begin{gathered}
\| \partial_{x}\left(V z^{(m)}\right)(\tau, y)-\partial_{x}\left(V z^{(m)}(\tau, \bar{y})\|\leq \widetilde{P}\| y-\bar{y} \|,\right. \\
\left\|\partial_{x}\left(V z^{(m)}\right)(\tau, y)\right\| \leq B+L\left(\|\varphi\|_{Y^{I}}+d_{0}+d_{1}\right),
\end{gathered}
$$

for $(\tau, y),(\tau, \bar{y}) \in H_{c}$. It follows from Assumptions $\mathrm{H}[f, \varphi]$ and $\mathrm{H}[V]$ that the terms

$$
\begin{aligned}
& \int_{0}^{t}\left\|\partial_{x} f\left(P\left[z^{(m)}, u\right](\xi, t, x)\right)-\partial_{x} f\left(P\left[z^{(m)}, u\right](\xi, \bar{t}, \bar{x})\right)\right\| d \xi \\
& \int_{0}^{t}\left|\partial_{p} f\left(P\left[z^{(m)}, u\right](\xi, t, x)\right)-\partial_{p} f\left(P\left[z^{(m)}, u\right](\xi, \bar{t}, \bar{x})\right)\right| d \xi
\end{aligned}
$$

can be estimated by

$$
\bar{d} \int_{0}^{t} \beta(\xi)\left\|g\left[z^{(m)}, u\right](\xi, t, x)-g\left[z^{(m)}, u\right](\xi, \bar{t}, \bar{x})\right\| d \xi .
$$


The above inequalities and Lemma 3.1 imply

$$
\begin{aligned}
\left\|G^{(m)}[u](t, x)-G^{(m)}[u](\bar{t}, \bar{x})\right\| \leq & P\left|\int_{t}^{\bar{t}} \widetilde{\gamma}(\xi) d \xi\right| \\
& +\Gamma(c)\left[\|x-\bar{x}\|+\left|\int_{t}^{\bar{t}}\|\gamma(\xi)\| d \xi\right|\right]
\end{aligned}
$$

on $H_{c}$. Conditions (25), (26) and (20)-(23) imply (24).

III. Now we prove that $G^{(m)}$ is a contraction on $C_{c}^{L}[s, \mu]$. If $u, \bar{u} \in C_{c}^{L}[s, \mu]$ then by Assumptions $\mathrm{H}[f, \varphi]$ and $\mathrm{H}[V]$ we get

$$
\left\|G^{(m)}[u](t, x)-G^{(m)}[\bar{u}](t, x)\right\| \leq(\Gamma(c)+P) \int_{0}^{t} \beta(\xi)\|u-\bar{u}\|_{\xi} d \xi
$$

for $(t, x) \in H_{c}$. For $u \in C_{c}^{L}[s, \mu]$ we put

$$
\|u\|_{\star}=\max \left\{\|u\|_{t} \exp \left[-2(\Gamma(c)+P) \int_{0}^{t} \beta(\tau) d \tau\right]: t \in[0, c]\right\} .
$$

Hence, by (27) we get

$$
\begin{aligned}
\| G^{(m)}[u]- & G^{(m)}[\bar{u}] \|_{t} \\
& \leq(\Gamma(c)+P)\|u-\bar{u}\|_{\star} \int_{0}^{t} \beta(\xi) \exp \left[2(\Gamma(c)+P) \int_{0}^{\xi} \beta(\tau) d \tau\right] d \xi \\
& \leq \frac{1}{2}\|u-\bar{u}\|_{\star} \exp \left[2(\Gamma(c)+P) \int_{0}^{t} \beta(\tau) d \tau\right], \quad t \in[0, a],
\end{aligned}
$$

and consequently

$$
\left\|G^{(m)}[u]-G^{(m)}[\bar{u}]\right\|_{\star} \leq \frac{1}{2}\|u-\bar{u}\|_{\star} .
$$

By the Banach fixed point theorem it follows that there is exactly one $u^{(m+1)} \in C_{c}^{L}[s, \mu]$ satisfying (18).

IV. Now we prove that the function $z^{(m+1)}: E \cup H_{c} \rightarrow \mathbb{R}$ given by (19) satisfies $\left(\mathrm{II}_{m+1}\right)$. Put

$$
\Delta(t, x, \bar{x})=z^{(m+1)}(t, \bar{x})-z^{(m+1)}(t, x)-\sum_{j=1}^{n} u_{j}^{(m+1)}(t, x)\left(\bar{x}_{j}-x_{j}\right)
$$

for $(t, x),(t, \bar{x}) \in H_{c}$. We prove that there exists $\widetilde{C} \in \mathbb{R}_{+}$such that

$$
|\Delta(t, x, \bar{x})| \leq \widetilde{C}\|x-\bar{x}\|^{2} \quad \text { for }(t, x),(t, \bar{x}) \in H_{c} .
$$


It follows from (19) and $\left(\mathrm{I}_{m+1}\right)$ that

$$
\begin{aligned}
\Delta(t, x, \bar{x})= & F\left[z^{(m)}, u^{(m+1)}\right](t, \bar{x})-F\left[z^{(m)}, u^{(m+1)}\right](t, x) \\
& -\sum_{j=1}^{n} G_{j}^{(m)}\left[u^{(m+1)}\right](t, x)\left(\bar{x}_{j}-x_{j}\right) .
\end{aligned}
$$

Write

$$
\begin{gathered}
g^{(m)}(\tau, t, x)=g\left[z^{(m)}, u^{(m+1)}\right](\tau, t, x), \\
P^{(m)}(\tau, t, x)=P\left[z^{(m)}, u^{(m+1)}\right](\tau, t, x), \\
Q^{(m)}(\xi, \tau, t, x, \bar{x})=\xi P^{(m)}(\tau, t, x)+(1-\xi) P^{(m)}(\tau, t, \bar{x}), \quad \xi \in[0,1] .
\end{gathered}
$$

Moreover we put

$$
\begin{aligned}
\Phi^{(m)}(t, x, \bar{x})= & \varphi\left(0, g^{(m)}(0, t, \bar{x})\right)-\varphi\left(0, g^{(m)}(0, t, x)\right) \\
& -\sum_{j=1}^{n} \partial_{x_{j}} \varphi\left(0, g^{(m)}(0, t, x)\right)\left(\bar{x}_{j}-x_{j}\right), \\
\Pi^{(m)}(t, x, \bar{x})= & \int_{0}^{t}\left[f\left(P^{(m)}(\tau, t, \bar{x})\right)-f\left(P^{(m)}(\tau, t, x)\right)\right] d \tau .
\end{aligned}
$$

It follows from (7) and (29) that

$$
\begin{aligned}
\Delta(t, x, \bar{x})= & \Phi^{(m)}(t, x, \bar{x})+\Pi^{(m)}(t, x, \bar{x}) \\
& -\sum_{j=1}^{n} \int_{0}^{t} \partial_{q_{j}} f\left(P^{(m)}(\tau, t, \bar{x})\right) u_{j}^{(m+1)}\left(\tau, g^{(m)}(\tau, t, \bar{x})\right) d \tau \\
& +\sum_{j=1}^{n} \int_{0}^{t} \partial_{q_{j}} f\left(P^{(m)}(\tau, t, x)\right) u_{j}^{(m+1)}\left(\tau, g^{(m)}(\tau, t, x)\right) d \tau \\
& -\sum_{j=1}^{n} \int_{0}^{t} \partial_{x_{j}} f\left(P^{(m)}(\tau, t, x)\right) d \tau\left(\bar{x}_{j}-x_{j}\right) \\
& -\sum_{j=1}^{n} \int_{0}^{t} \partial_{p} f\left(P^{(m)}(\tau, t, x)\right) \partial_{x_{j}}\left(V z^{(m)}\right)\left(\tau, g^{(m)}(\tau, t, x)\right) d \tau\left(\bar{x}_{j}-x_{j}\right) .
\end{aligned}
$$

By the Hadamard mean value theorem we have

$$
\begin{aligned}
& \Pi^{(m)}(t, x, \bar{x}) \\
& =\int_{0}^{t} \sum_{j=1}^{n} \int_{0}^{1} \partial_{x_{j}} f\left(Q^{(m)}(\xi, \tau, t, x, \bar{x})\right) d \xi\left(g_{j}^{(m)}(\tau, t, \bar{x})-g_{i}^{(m)}(\tau, t, x)\right) d \tau
\end{aligned}
$$




$$
\begin{aligned}
& +\int_{0}^{t} \int_{0}^{1} \partial_{p} f\left(Q^{(m)}(\xi, \tau, t, x, \bar{x})\right) d \xi \\
& \times\left[\left(V z^{(m)}\right)\left(\tau, g^{(m)}(\tau, t, \bar{x})\right)-\left(V z^{(m)}\right)\left(\tau, g^{(m)}(\tau, t, x)\right)\right] d \tau \\
& +\int_{0}^{t} \sum_{j=1}^{n} \int_{0}^{1} \partial_{q_{j}} f\left(Q^{(m)}(\xi, \tau, t, x, \bar{x})\right) d \xi \\
& \times\left[u_{j}^{(m+1)}\left(\tau, g^{(m)}(\tau, t, \bar{x})\right)-u_{j}^{(m+1)}\left(\tau, g^{(m)}(\tau, t, x)\right)\right] d \tau .
\end{aligned}
$$

Write

$$
\Lambda^{(m)}=\left(\Lambda_{1}^{(m)}, \ldots, \Lambda_{n}^{(m)}\right)
$$

where

$$
\Lambda_{j}^{(m)}(\tau, t, x, \bar{x})=\int_{\tau}^{t}\left[\partial_{q_{j}} f\left(P^{(m)}(\xi, t, \bar{x})\right)-\partial_{q_{j}} f\left(P^{(m)}(\xi, t, x)\right)\right] d \xi, \quad 1 \leq j \leq n .
$$

Then

$$
g^{(m)}(\tau, t, \bar{x})-g^{(m)}(\tau, t, x)-(\bar{x}-x)=\Lambda^{(m)}(\tau, t, x, \bar{x}) .
$$

V. To simplify the formulation of the next properties of the function $\Delta$ we define

$$
\begin{aligned}
\bar{\Delta}(t, x, \bar{x}) & =\bar{\Phi}^{(m)}(t, x, \bar{x}) \\
& +\int_{0}^{t} \sum_{j=1}^{n} \int_{0}^{t}\left[\partial_{x_{j}} f\left(Q^{(m)}(\xi, \tau, t, x, \bar{x})\right)-\partial_{x_{j}} f\left(P^{(m)}(\tau, t, x)\right)\right] d \xi \\
& \times\left[g_{j}^{(m)}(\tau, t, \bar{x})-g_{j}^{(m)}(\tau, t, x)\right] d \tau \\
& +\int_{0}^{t}\left[\partial_{p} f\left(Q^{(m)}(\xi, \tau, t, x, \bar{x})\right)-\partial_{p} f\left(P^{(m)}(\tau, t, x)\right)\right] d \xi \\
& \times\left[\left(V z^{(m)}\right)\left(\tau, g^{(m)}(\tau, t, \bar{x})\right)-\left(V z^{(m)}\right)\left(\tau, g^{(m)}(\tau, t, x)\right)\right] d \tau \\
& +\int_{0}^{t} \sum_{j=1}^{n} \int_{0}^{1}\left[\partial_{q_{j}} f\left(Q^{(m)}(\xi, \tau, t, x, \bar{x})\right)-\partial_{q_{j}} f\left(P^{(m)}(\tau, t, \bar{x})\right)\right] d \xi \\
& \times\left[u_{j}^{(m+1)}\left(\tau, g^{(m)}(\tau, t, \bar{x})\right)-u_{j}^{(m+1)}\left(\tau, g^{(m)}(\tau, t, x)\right)\right] d \tau \\
& +\int_{0}^{t} \partial_{p} f\left(P^{(m)}(\tau, t, x)\right) \Theta^{(m)}(\tau, t, x, \bar{x}) d \tau,
\end{aligned}
$$

where 


$$
\begin{aligned}
\bar{\Phi}^{(m)}(t, x, \bar{x})= & \varphi\left(0, g^{(m)}(0, t, \bar{x})\right)-\varphi\left(0, g^{(m)}(0, t, x)\right) \\
& -\sum_{j=1}^{n} \partial_{x_{j}} \varphi\left(0, g^{(m)}(0, t, x)\right)\left[g_{j}^{(m)}(0, t, \bar{x})-g_{j}^{(m)}(0, t, x)\right], \\
\Theta^{(m)}(\tau, t, x, \bar{x})= & \left(V z^{(m)}\right)\left(\tau, g^{(m)}(\tau, t, \bar{x})\right)-\left(V z^{(m)}\right)\left(\tau, g^{(m)}(\tau, t, x)\right) \\
& -\sum_{j=1}^{n} \partial_{x_{j}}\left(V z^{(m)}\right)\left(\tau, g^{(m)}(\tau, t, x)\right)\left[g_{j}^{(m)}(\tau, t, \bar{x})-g_{j}^{(m)}(\tau, t, x)\right] .
\end{aligned}
$$

We define the function $\widetilde{\Delta}$ by

$$
\widetilde{\Delta}(t, x, \bar{x})=\sum_{j=1}^{n} \partial_{x_{j}} \varphi\left(0, g^{(m)}(0, t, x)\right) \Lambda_{j}^{(m)}(0, t, x, \bar{x})
$$$$
+\sum_{j=1}^{n} \int_{0}^{t} \partial_{x_{j}} f\left(P^{(m)}(\tau, t, x)\right) \Lambda_{j}^{(m)}(\tau, t, x, \bar{x}) d \tau
$$$$
+\sum_{j=1}^{n} \int_{0}^{t} \partial_{p} f\left(P^{(m)}(\tau, t, x)\right) \partial_{x_{j}}\left(V z^{(m)}\right)\left(\tau, g^{(m)}(\tau, t, x)\right) \Lambda_{j}^{(m)}(\tau, t, x, \bar{x}) d \tau
$$$$
-\sum_{j=1}^{n} \int_{0}^{t}\left[\partial_{q_{j}} f\left(P^{(m)}(\tau, t, \bar{x})\right)-\partial_{q_{j}} f\left(P^{(m)}(\tau, t, x)\right)\right] u_{j}^{(m+1)}\left(\tau, g^{(m)}(\tau, t, x)\right) d \tau .
$$

Then $\Delta(t, x, \bar{x})=\bar{\Delta}(t, \bar{x})+\widetilde{\Delta}(t, x, \bar{x})$.

VI. Now we prove that

$$
\widetilde{\Delta}(t, x, \bar{x})=0 \quad \text { for }(t, x),(t, \bar{x}) \in H_{c} .
$$

Write $\Xi^{(m)}=\left(\Xi_{1}^{(m)}, \ldots, \Xi_{n}^{(m)}\right)$, where

$$
\begin{aligned}
\Xi_{j}^{(m)}(\xi, t, x) & =\partial_{x_{j}} \varphi\left(0, g^{(m)}(0, t, x)\right) \\
& +\int_{0}^{\xi} \partial_{p} f\left(P^{(m)}(\tau, t, x)\right) \partial_{x_{j}}\left(V z^{(m)}\right)\left(\tau, g^{(m)}(\tau, t, x)\right) d \tau \\
& +\int_{0}^{\xi} \partial_{x_{j}} f\left(P^{(m)}(\tau, t, x)\right) d \tau-u_{j}^{(m+1)}\left(\xi, g^{(m)}(\xi, t, x)\right), \quad 1 \leq j \leq n .
\end{aligned}
$$

It follows from (31) that

$$
\widetilde{\Delta}(t, x, \bar{x})=\sum_{j=1}^{n} \int_{0}^{t} \Xi_{j}^{(m)}(\xi, t, x)\left[\partial_{q_{j}} f\left(P^{(m)}(\xi, t, \bar{x})\right)-\partial_{q_{j}} f\left(P^{(m)}(\xi, t, x)\right)\right] d \xi .
$$

Since

$$
g^{(m)}\left(\xi, \tau, g^{(m)}(\tau, t, x)\right)=g^{(m)}(\xi, t, x)
$$


and $u^{(m+1)}$ satisfies equation (18) on $H_{c}$ it follows that

$$
\Xi^{(m)}(\xi, t, x)=0 \quad \text { for }(t, x) \in H_{c}, \xi \in[0, t],
$$

and consequently $\widetilde{\Delta}(t, x, \bar{x})=0$. Thus we have proved that

$$
\Delta(t, x, \bar{x})=\bar{\Delta}(t, x, \bar{x}) \quad \text { for }(t, x),(t, \bar{x}) \in H_{c} .
$$

An easy computation shows that

$$
\left|\bar{\Phi}^{(m)}(t, x, \bar{x})\right| \leq r_{2}\left\|g^{(m)}(\tau, t, \bar{x})-g^{(m)}(\tau, t, x)\right\|^{2}
$$

and

$$
\begin{aligned}
& \left|\left(V z^{(m)}\right)\left(\tau, g^{(m)}(\tau, t, \bar{x})\right)-\left(V z^{(m)}\right)\left(\tau, g^{(m)}(\tau, t, x)\right)\right| \\
& \quad \leq\left[L\left(\|\varphi\|_{Y^{I}}+d_{0}+d_{1}\right)+B\right]\left\|g^{(m)}(\tau, t, \bar{x})-g^{(m)}(\tau, t, x)\right\| .
\end{aligned}
$$

Moreover we have

$$
\begin{aligned}
&\left\|u^{(m+1)}\left(\tau, g^{(m)}(\tau, t, \bar{x})\right)-u^{(m+1)}\left(\tau, g^{(m)}(\tau, t, x)\right)\right\| \\
& \leq s_{1}\left\|g^{(m)}(\tau, t, \bar{x})-g^{(m)}(\tau, t, x)\right\|,
\end{aligned}
$$

and

$$
\begin{aligned}
& \left|\Theta^{(m)}(\tau, t, x, \bar{x})\right| \\
& \quad \leq\left[M\left(\|\varphi\|_{Y^{I+L}}+d_{0}+d_{1}+d_{2}\right)+C\right]\left\|g^{(m)}(\tau, t, \bar{x})-g^{(m)}(\tau, t, x)\right\|^{2} .
\end{aligned}
$$

The above estimates and (30), (33) and Lemma 3.1 imply (28) for some $\widetilde{C} \in \mathbb{R}_{+}$and consequently

$$
\partial_{x} z^{(m+1)}(t, x)=u^{(m+1)}(t, x) \quad \text { on } H_{c} .
$$

Thus condition $\left(\mathrm{II}_{m+1}\right)$ is proved.

VII. Now we prove that $z^{(m+1)} \in C_{\varphi \cdot c}^{I+L}[d, \lambda]$. Of course $z^{(m+1)}$ is continuous on $H_{c}$ and $z^{(m+1)}=\varphi$ on $E$. Moreover from $\left(\mathrm{II}_{m+1}\right)$ it follows that

$$
\begin{gathered}
\left\|\partial_{x} z^{(m+1)}(t, x)\right\| \leq d_{1} \\
\left\|\partial_{x} z^{(m+1)}(t, x)-\partial_{x} z^{(m+1)}(\bar{t}, \bar{x})\right\| \leq\left|\int_{t}^{\bar{t}} \lambda_{1}(\xi) d \xi\right|+d_{2}\|x-\bar{x}\|
\end{gathered}
$$

on $H_{c}$. Our assumptions and (22), (23) also imply the estimates

$$
\left|z^{(m+1)}(t, x)\right| \leq d_{0}, \quad\left|z^{(m+1)}(\bar{t}, x)-z^{(m+1)}(t, x)\right| \leq\left|\int_{t}^{\bar{t}} \lambda_{0}(\xi) d \xi\right|
$$

on $H_{c}$, which completes the proof of $\left(\mathrm{I}_{m+1}\right)$. Thus Theorem 5.1 follows by induction. 
6. Convergence of the sequences $\left\{z^{(m)}\right\}$ and $\left\{u^{(m)}\right\}$. Now we prove that the sequences $\left\{z^{(m)}\right\}$ and $\left\{u^{(m)}\right\}$ are uniformly convergent on $H_{c}$ if the constant $c$ is sufficiently small. Write

$$
\bar{\Gamma}(t)=\Gamma(t)+P \max \left\{1, L_{0}\right\},
$$

and

$$
\begin{aligned}
\Theta(t) & =\exp \left[\bar{\Gamma}(c) \int_{0}^{c} \beta(\xi) d \xi\right]\{L \widetilde{\gamma}(t)+\bar{\Gamma}(c) \beta(t)\}, \\
\Theta_{0}(t) & =L_{0}[\widetilde{\gamma}(t)+\widetilde{\Gamma}(c) \beta(t)]+s_{0} \beta(t) \max \left\{1, L_{0}\right\}, \\
\widetilde{\Theta}(t) & =\Theta_{0}(t)+\widetilde{C} \Theta(t), \quad \widetilde{C}=\int_{0}^{c}[\widetilde{\Gamma}(c) \beta(\xi)+2\|\gamma(\xi)\|] d \xi
\end{aligned}
$$

TheOREM 6.1. Suppose that

1) Assumptions $\mathrm{H}[X], \mathrm{H}[V], \mathrm{H}[f, \varphi]$ are satisfied and $\varphi \in Y^{I+L}$,

2) the constants $d=\left(d_{0}, d_{1}, d_{2}\right) \in \mathbb{R}_{+}^{3}, s=\left(s_{0}, s_{1}\right) \in \mathbb{R}_{+}^{2}, c \in(0, a]$ and the functions $\lambda=\left(\lambda_{0}, \lambda_{1}\right), \lambda_{0}, \lambda_{1}, \mu \in L\left([0, c], \mathbb{R}_{+}\right)$satisfy the conditions (20)-(23),

3) $c \in(0, a]$ is a constant so small that

$$
\int_{0}^{c}[\Theta(\tau)+\widetilde{\Theta}(\tau)] d \tau<1 .
$$

Then the sequences $\left\{z^{(m)}\right\}$ and $\left\{u^{(m)}\right\}$ are uniformly convergent on $H_{c}$.

Proof. We first prove that

$$
\begin{aligned}
& \left\|u^{(m+1)}-u^{(m)}\right\|_{t} \leq \int_{0}^{t} \Theta(\xi)\left[\left\|z^{(m)}-z^{(m-1)}\right\|_{\xi}+\left\|u^{(m)}-u^{(m-1)}\right\|_{\xi}\right] d \xi \\
& \left\|z^{(m+1)}-z^{(m)}\right\|_{t} \leq \int_{0}^{t} \widetilde{\Theta}(\xi)\left[\left\|z^{(m)}-z^{(m-1)}\right\|_{\xi}+\left\|u^{(m)}-u^{(m-1)}\right\|_{\xi}\right] d \xi
\end{aligned}
$$

for $t \in[0, c]$. We conclude from Assumptions $\mathrm{H}\left[\partial_{q} f\right], \mathrm{H}[f, \varphi], \mathrm{H}[V]$ and from Lemma 3.1 that

$$
\begin{aligned}
& \left\|u^{(m+1)}-u^{(m)}\right\|_{t} \leq \bar{\Gamma}(c) \int_{0}^{t} \beta(\xi)\left\|u^{(m+1)}-u^{(m)}\right\|_{\xi} d \xi \\
& \quad+\int_{0}^{t}[\bar{\Gamma}(c) \beta(\xi)+L \widetilde{\gamma}(\xi)]\left\|z^{(m)}-z^{(m-1)}\right\|_{\xi} d \xi+L \int_{0}^{t} \widetilde{\gamma}(\xi)\left\|u^{(m)}-u^{(m-1)}\right\|_{\xi} d \xi
\end{aligned}
$$

for $t \in[0, c]$. Hence, using the Gronwall inequality and (34), we get (38). 
It follows from (19) and from Lemma 3.1 that

$$
\begin{aligned}
\left\|z^{(m+1)}-z^{(m)}\right\|_{t} \leq & \int_{0}^{t} \Theta_{0}(\xi)\left[\left\|z^{(m)}-z^{(m-1)}\right\|_{\xi}+\left\|u^{(m)}-u^{(m-1)}\right\|_{\xi}\right] d \xi \\
& +\int_{0}^{t}[\widetilde{\Gamma}(c) \beta(\xi)+2\|\gamma(\xi)\|]\left\|u^{(m+1)}-u^{(m)}\right\|_{\xi} d \xi, \quad t \in[0, c] .
\end{aligned}
$$

Now, from (38) and (35), (36), we obtain (39). According to (38) and (39), we have

$$
\begin{aligned}
&\left\|z^{(m+1)}-z^{(m)}\right\|_{t}+\left\|u^{(m+1)}-u^{(m)}\right\|_{t} \\
& \quad \leq \int_{0}^{t}[\Theta(\xi)+\widetilde{\Theta}(\xi)]\left[\left\|z^{(m)}-z^{(m-1)}\right\|_{\xi}+\left\|u^{(m)}-u^{(m-1)}\right\|_{\xi}\right] d \xi
\end{aligned}
$$

for $t \in[0, c], m \geq 1$, and there exists $\delta \in(0,1)$ such that

$$
\begin{aligned}
& \left\|z^{(m+1)}-z^{(m)}\right\|_{t}+\left\|u^{(m+1)}-u^{(m)}\right\|_{t} \\
& \quad \leq \delta\left[\left\|z^{(m)}-z^{(m-1)}\right\|_{t}+\left\|u^{(m)}-u^{(m-1)}\right\|_{t}\right] .
\end{aligned}
$$

From (16), (17) and from Theorem 5.1 it follows that there is $C^{\star} \in \mathbb{R}_{+}$such that

$$
\left\|z^{(1)}-z^{(0)}\right\|_{t}+\left\|u^{(1)}-u^{(0)}\right\|_{t} \leq C^{\star}, \quad t \in[0, c] .
$$

Then the uniform convergence of the sequences $\left\{z^{(m)}\right\}$ and $\left\{u^{(m)}\right\}$ on $H_{c}$ follows from (40).

7. Existence of solutions of initial value problems. Now we give the main existence result for problem (1), (2).

Theorem 7.1. Suppose that

1) $\varphi \in Y^{I+L}$ and Assumptions $\mathrm{H}[X], \mathrm{H}[V], \mathrm{H}[f, \varphi]$ are satisfied,

2) conditions (20)-(23) and (37) hold.

Then there exists a solution $v: E \cup H_{c} \rightarrow \mathbb{R}$ of problem (1), (2). Moreover $v \in C_{\varphi . c}^{I+L}[d, \lambda]$ and $u \in C_{c}^{L}[s, \mu]$, where $u=\left.\partial_{x} v\right|_{H_{c}}$. If $\widetilde{\varphi} \in Y^{I+L}$ and $\widetilde{v} \in C_{\widetilde{\varphi} . c}^{I+}[d, \lambda]$ is a solution of equation (1) with the initial condition

$$
z(t, x)=\widetilde{\varphi}(t, x) \quad \text { on } E,
$$

then there exists $\kappa \in C\left([0, c], \mathbb{R}_{+}\right)$such that

$$
\left\|\left.(v-\widetilde{v})\right|_{H_{t}}\right\|_{t}+\left\|\left.\left(\partial_{x} v-\partial_{x} \widetilde{v}\right)\right|_{H_{t}}\right\|_{t} \leq \kappa(t)\|\varphi-\widetilde{\varphi}\|_{Y^{I}}, \quad t \in[0, c] .
$$

Proof. From Theorem 6.1 it follows that there exist functions $v \in$ $C_{\varphi . c}^{I+L}[d, \lambda]$ and $u \in C_{c}^{L}[s, \mu]$ such that $\left\{z^{(m)}\right\}$ converges uniformly to $v$ and $\left\{u^{(m)}\right\}$ converges uniformly to $u$ on $H_{c}$. Moreover $\partial_{x} v$ exists on $H_{c}$ and 
$\partial_{x} v=u$. Thus we get

$$
\begin{aligned}
& v(t, x)=\varphi\left(0, g\left[v, \partial_{x} v\right](0, t, x)\right)+\int_{0}^{t} f\left(P\left[v, \partial_{x} v\right](\tau, t, x)\right) d \tau \\
& -\sum_{k=1}^{n} \int_{0}^{t} \partial_{g_{k}} f\left(P\left[v, \partial_{x} v\right](\tau, t, x)\right) \partial_{x_{k}} v\left(\tau, g\left[v, \partial_{x} v\right](\tau, t, x)\right) d \tau, \quad(t, x) \in H_{c}
\end{aligned}
$$

and

$$
g\left[v, \partial_{x} v\right](\tau, t, x)=x+\int_{\tau}^{t} \partial_{q} f\left(P\left[v, \partial_{x} v\right](\tau, t, x)\right) d \tau, \quad(t, x) \in H_{c} .
$$

For a given $(t, x) \in H_{c}$, put $y=g\left[v, \partial_{x} v\right](0, t, x)$ and $Q(\tau, y)$

$$
=\left(\tau, g\left[v, \partial_{x} v\right](\tau, 0, y),(V v)\left(\tau, g\left[v, \partial_{x} v\right](\tau, 0, y)\right), \partial_{x} v\left(\tau, g\left[v, \partial_{x} v\right](\tau, 0, y)\right)\right) .
$$

Then the statement (42) on $H_{c}$ is equivalent to

$$
\begin{aligned}
v\left(\tau, g\left[v, \partial_{x} v\right](\tau, 0, y)\right) & =\varphi(0, y)+\int_{0}^{t} f(Q(\tau, y)) d \tau \\
& -\sum_{k=1}^{n} \int_{0}^{t} \partial_{q_{k}} f(Q(\tau, y)) \partial_{x_{k}} v\left(\tau, g\left[v, \partial_{x} v\right](\tau, 0, y)\right) d \tau
\end{aligned}
$$

The relations

$$
y=g\left[v, \partial_{x} v\right](0, t, x) \quad \text { and } \quad x=g\left[v, \partial_{x} v\right](t, 0, y)
$$

are equivalent for $(t, x) \in H_{c}, y \in[-b, b]$. By differentiating (43) with respect to $t$ and by putting again $x=g\left[v, \partial_{x}\right](t, 0, y)$, we conclude that $v$ satisfies (1) for almost all $t \in I[x]$ for fixed $x \in[-b, b]$. It is clear that $v$ satisfies the initial condition (2).

Now we prove (41). The functions $\left(v, \partial_{x} v\right)$ satisfy the system of integral functional equations $(9),(10)$ and $\left(\widetilde{v}, \partial_{x} \widetilde{v}\right)$ is a solution of the corresponding system with $\widetilde{\varphi}$ instead of $\varphi$. Hence we conclude that there exist a function $\bar{\lambda} \in L\left([0, c], \mathbb{R}_{+}\right)$and a constant $\bar{d} \in \mathbb{R}_{+}$such that the function

$$
\Psi(t)=\left\|\left.(v-\widetilde{v})\right|_{H_{t}}\right\|_{t}+\left\|\left.\left(\partial_{x} v-\partial_{x} \widetilde{v}\right)\right|_{H_{t}}\right\|_{t}
$$

satisfies the integral inequality

$$
\Psi(t) \leq \bar{d}\|\varphi-\widetilde{\varphi}\|_{Y^{I}}+\int_{0}^{t} \bar{\lambda}(\tau) \Psi(\tau) d \tau, \quad t \in[0, c] .
$$

Using the Gronwall inequality we obtain (41) with

$$
\kappa(t)=\bar{d} \exp \left[\int_{0}^{t} \Psi(\tau) d \tau\right] .
$$

This proves the theorem. 
REMARK 7.2. If all the assumptions of Theorem 7.1 are satisfied and the functions

$$
f(\cdot, x, p, q), \quad \partial_{x} f(\cdot, x, p, q), \quad \partial_{p} f(\cdot, x, p, q), \quad \partial_{q} f(\cdot, x, p, q)
$$

are continuous on $I[x]$ for $(x, p, q) \in[-b, b] \times \mathbb{R} \times \mathbb{R}^{n}$ then we get classical solutions of equation (1).

REMARK 7.3. The existence result presented in this paper is new also in the case when the initial set is bounded. Differential equations with deviated variables and differential integral equations can be obtained by specializing the operator $V$.

\section{References}

[1] P. Brandi and C. Marcelli, Haar inequality in hereditary setting and applications, Rend. Sem. Mat. Univ. Padova 96 (1996), 177-194.

[2] —, - On mixed problem for first order partial differential functional equations, Atti Sem. Mat. Fis. Univ. Modena 46 (1998), 497-510.

[3] S. Burys, On partial differential functional inequalities of the first order, Univ. Iagel. Acta Math. 16 (1974), 107-112.

[4] L. Byszewski, Finite systems of strong nonlinear differential functional degenerate implicit inequalities with first order partial derivatives, ibid. 29 (1992), 77-84.

[5] M. Cinquini Cibrario, Sopra una classe di sistemi di equazioni non lineari a derivate parziali in più variabili indipendenti, Ann. Mat. Pura Appl. 140 (1985), 223-253.

[6] T. Duc Van, M. Tsuji and N. Duy Thai Son, The Characteristic Method and Its Generalizations for First Order Nonlinear Partial Differential Equations, Chapman \& Hall, Boca Raton and New York, 2000.

[7] Y. Hino, S. Murakami and T. Naito, Functional Differential Equations with Unbounded Delay, Springer, Berlin, 1991.

[8] Z. Kamont, Hyperbolic Functional Differential Inequalities and Applications, Kluwer, Dordrecht, 1999.

[9] - On the local Cauchy problem for Hamilton Jacobi equations with a functional dependence, Rocky Mountain J. Math. 30 (2000), 587-608.

[10] Z. Kowalski, A difference method for certain hyperbolic systems of non-linear partial differential equations of the first order, Bull. Acad. Polon. Sci. Sér. Sci. Math. Astronom. Phys. 16 (1968), 297-302.

[11] Y. Lakshmikantham and S. Leela, Differential and Integral Inequalities, Academic Press., New York and London, 1969.

[12] V. Lakshmikantham, L. Z. Wen and B. G. Zhang, Theory of Differential Equations with Unbounded Delay, Kluwer, Dordrecht, 1994.

[13] A. Nadolski, Functional differential problems for quasilinear hyperbolic systems with unbounded delay, Funct. Differ. Equ. 9 (2002), 227-246.

[14] A. Pliś, On difference inequalities corresponding to partial differential inequalities of first order, Ann. Polon. Math. 20 (1968), 179-181.

[15] J. Szarski, Characteristics and Cauchy problem for nonlinear partial differential equations of first order, University of Kansas, Lawrence, Kansas, 1959.

[16] —, Differential Inequalities, Polish Sci. Publ., Warszawa, 1967. 
[17] T. Ważewski, Sur l'appréciation de domaine d'existence des intégrales de l'équation aux dérivées partielles du premier ordre, Ann. Soc. Polon. Math. 14 (1935), 149-175.

[18] K. Zima, Sur les équations aux dérivées partielles du premier ordre à argument fonctionnel, Ann. Polon. Math. 22 (1969), 49-59.

Foundations of Informatics Department

Gdańsk University of Technology

Narutowicza 11/12

80-952 Gdańsk, Poland

E-mail: nadolski@eti.pg.gda.pl 